\title{
In vitro Testing of Experimental and Commercial Bracket Bonding Materials
}

\author{
ANCA S. MESAROS ${ }^{1}$, CRISTIAN ROMANEC ${ }^{2 *}$, MICHAELA MESAROS ${ }^{3}$, MARIOARA MOLDOVAN ${ }^{4}$, IOANA BALDEA $^{5}$ \\ ${ }^{1}$ luliu Hatieganu University of Medicine and Pharmacy, Department of Dental Propaedeutics and Esthetics, 32 Clinicilor Str., \\ 400006, Cluj Napoca, Romania \\ ${ }^{2}$ Grigore T. Popa University of Medicine and Pharmacy, Department of Orthodontics, 16 Universitatii Str., 700115, Iasi, Romania \\ ${ }^{3}$ Iuliu Hatieganu University of Medicine and Pharmacy, Department of Paedriatic Dentistry, 33 Motilor Str., 400001, Cluj Napoca, \\ Romania \\ ${ }^{4}$ Raluca Ripan Institute of Chemistry, Babes Bolyai University, 20 Fatanele Str., 400294, Cluj Napoca, Romania \\ ${ }^{5}$ Iuliu Hatieganu University of Medicine and Pharmacy, Department of Physiology, 3 Clinicilor Str. Str., 400006, Cluj Napoca, \\ Romania
}

\begin{abstract}
The aims of our study were to assess the cytotoxic effect of five orthodontic bonding materials, in vitro, on HUVECs to study the induction of apoptosis and inflammatory response generated to assess the shear-bond strength of the 5 tested materials in order to quantify their performance. Standardized samples from each material were obtained and incubated with HUVEC cells for 24 and $72 \mathrm{~h}$ immersed in complete medium. Cell viability was determined by means of MTS method. Active caspase 3 and TNF $\alpha$ protein levels were measured through ELISA techniques. The shear-bond strength was tested on 60 extracted premolars which were bonded with the same type of bracket, using the 5 different materials. Statistical analysis Student T-tests, Chi-square and Anova tests were used for results interpretation. Cell viability was decreased with material exposure in a time dependent manner. All materials exerted cytotoxic effects, the experimental materials showed a significantly higher decrease in cell viability at the $72 \mathrm{~h}$ reading. Shear Bond strength was superior for the resin commercial bracket-bonding materials. The study shows thatorthodontic adhesives' cytotoxicity and physical performance is related to their chemical properties and proves that all orthodontic practitioners should use freely their material of choice on condition they are aware of all its' properties.
\end{abstract}

Keywords: orthodontic bonding materials, apoptosis, inflammatory response, MTS method, ELISA techniques

Developments of orthodontic adhesives are continuous but their biocompatibility is an important issue for orthodontists. Studies present in the literature nowadays is either focusing on the biocompatibility, or the mechanical properties of commercially available orthodontic adhesives and very little research has been done in corroborating both aspects. Several studies from the literature have investigated the biocompatibility of orthodontic bonding materials and most of them concluded that the adhesives are cytotoxic to some degree [1]. They are responsible for hypersensitivity and allergic reactions, which in a chain reaction can lead to systemic involvement [2-4]. The ideal orthodontic adhesive should have good chemical and physical properties, be no irritating to surrounding tissue and be easy to manage [5]. Orthodontic adhesives are used to glue orthodontic attachments directly to the surface of the tooth. There are two types of adhesive material applied in orthodontic attachmentbonding: resin base and resin hybrid glass ionomer base adhesives, but in both categories there is a large variety of commercial products with different physical properties. Some adhesives make direct contact with softoral tissues and may cause irritation. $[3,6]$ In orthodontic treatments, controlling periodontal tissue health is important. It is hypothesized that the orthodontic adhesives can induce gingival inflammation. Besides knowing the potential cytotoxic effect that a material might have, the physical performance of such a material should also be known in order for the orthodontist to choose wisely the best materials to work with.

In this study we evaluated the cytotoxic effects of 3 commercial orthodontic bonding materials, 1 flowable selfetching commercial composite and 1 new experimental resin modified glass-ionomer, in vitro, on human umbilical endothelial vein cultures (HUVEC). We also studied the induction of apoptosis and inflammatory response generated by these compounds, in an effort to find the pathological mechanism involved in generation of the cytotoxicity in vitro, as well as the clinical side effects. The physical performance of the materials was assessed by Shear-Bond Strength testing in standardized conditions.

\section{Experimental part}

Materials and methods

The adhesives used in the present study were:

- the resin base adhesive Light Bond (Reliance Orthodontic Products, Itasca, II)

- the resin base adhesive Opal Bond MV (Opal Orthodontics, Ultradent)

- the resin self-etching composite Vertise (Kerr) - a commercial material with restorative indications, tested to search an eventual indication in bracket boding $\mathrm{GC})$

- the resin hybrid glass ionomer adhesive Fuji Ortho LC (

- an experimental resin hybrid glass ionomer material developed at the Raluca Ripan Institute of Chemistry (UBB)

The experimental resin hybrid glass-ionomer is a new chemical formula developed by the researchers from the Raluca Ripan Institute of Chemistry, for which the present testing was the first performance test.

\section{In vitro testing}

Bonding material sample preparation: Silicone moulds of a cylindrical metallic bar with dimensions of $4 \times 4 \mathrm{~mm}$ were filled with orthodontic bonding material. 8 material 
samples of each bonding and experimental material were obtained after polymerization of $40 \mathrm{~s}$ at each end of the mould, according to the manufacture's instructions. Lightcuring was performed using the Bluephase C8 lamp from IVOCLAR VIVADENT (Schaan Principality of Liechtenstein) avoiding air exposure. No grinding or polishing was performed on the resin samples. The sample thickness was measured in three areas using a digital calliper with an accuracy of $0.01 \mathrm{~mm}$.

Cell source: Commercial human umbilical vein endothelial cells (HUVEC) were bought from European Collection of Cell Cultures (ECACC, Porton Down, and Salisbury, UK) and multiplied in standard medium: RPMI, supplemented with $10 \%$ fetal calf serum (FCS), gentamicin $50 \mu \mathrm{g} / \mathrm{mL}$, amphotericin $100 \mu \mathrm{g} / \mathrm{ml}$ (Biochrom AG, Germany). Cell cultures in the $23^{\text {rd }}$ and $26^{\text {th }}$ passage were used.

Cell viability testing was done using CellTiter $96 \AA$ AQueous Non-Radioactive Cell Proliferation Assay (Promega Corporation, Madison, USA). HUVEC cultures seeded at a density of $10^{4} /$ well in ELISA 96 wells micro titration flat bottom plaques (TPP, Switzerland), were allowed to settle for $24 \mathrm{~h}$ in medium, then exposed to biomaterial samples immersed in complete medium for 24 and $72 \mathrm{~h}$; afterwards washed and settled for $24 \mathrm{~h}$ in complete medium. Cells were then treated with $20 \mu \mathrm{L}$ of [3-(4,5-dimethylthiazol-2-yl)-5-(3-carboxymethoxyphenyl) -2-(4-sulfophenyl)-2H-tetrazolium, inner salt MTS/ (phenazine methosulfate) PMS mixture in $100 \mu \mathrm{L}$ complete medium for $2 \mathrm{~h}$. Absorbance was read at $490 \mathrm{~nm}$, using an ELISA plate reader (Tecan, Switzerland). HUVEC cultures exposed only to medium were used as controls. Chemically inert glass samples with the same dimensions as the ones from the tested materials were used for the control cultures. The experiments were performed in triplicate. The number of cells was estimated, being directly correlated with the absorbance and the results are presented as \% of initial value.

Microscopy: The Annexin V-fluorescein isothiocyanate (FITC) apoptosis detection kit was used in conjunction with the vital dye propidium iodide (PI) to identify late apoptotic (necrotic) cells (penetrated by PI); the kit was also used to identify viable cells (Annexin V-FITC negative) with intact cellular membranes as well as early apoptotic cells (Annexin V-FITC positive) which were not penetrated by $\mathrm{PI}$ [8]. HUVEC cultures were seeded on glass chamber slides (Nalgene, Rochester, NY, USA) at a density of $5 \times 10^{4} /$ $\mathrm{cm}^{2}$ as previously described [8], then treated as described above (at viability testing). Cells were stained with Annexin V-FITC and PI according to the manufacturer's instructions and then fixed in $2 \%$ paraformaldehyde. Pictures were taken using a Zeiss LSM 710 confocal laser microscope. The number of cells positive or negative for Annexin $V$ and $\mathrm{PI}$ was counted for each sample, and cells were divided in the following two categories: (1) viable cells: Annexin $\mathrm{V}$ $(-) / P I(-) ;(2)$ apoptotic cells: Annexin V (+)/PI (-) (in early apoptosis), and Annexin V $(+) / \mathrm{PI}(+)$ (in late apoptosis). At least 200 cells per sample were scored by eye, at a magnification of 63x. The experiments were performed in triplicate.

ELISA: HUVEC cultures seeded at a density of $10^{6} /$ well in 6 well plates (TPP, Switzerland), were treated as described above (at viability testing). The human Quantikine Active Caspase-3 and TNF $\alpha$ Immunoassay kits from R\&D Systems, Inc (USA) were used. Cell extracts and, respectively supernatants were treated as indicated by the producer, readings were done at $450 \mathrm{~nm}$ with correction wavelength set at $540 \mathrm{~nm}$, using an ELISA plate reader (Tecan, Switzerland)[8-11].

\section{Shear-Bond Strength testing}

60 fresh human premolars ( 30 mandibular and 30 from the maxilla) were selected from extracted teeth from patients aged 16 to 25 years and stored in a solution of artificial saliva. The storage time varied from 8 to 11 weeks. Tooth selection criteria included those with intactenamel, no pretreatment with chemical agents (for example, hydrogen peroxide), no cracks, no lesions from the extraction forceps, and no caries or restorations. These teeth were extracted for orthodontic purposes and all patients agreed to donate their extracted teeth for science experiments. After professional cleaning procedures, teeth were embedded in acrylic resin with a label bearing the code of each sample and only the crowns of the teeth remained exposed.

The specimens were divided into 5 groups $(n=12)$ according to the material used for bracket bonding. The selection of teeth within each group was randomized but each group had 6 upper and 6 mandibular premolars.

60 standard edgewise stainless steel premolar brackets $(0.022 \times 0.028$ inch slot) (GAC Orthodontic Products, New York, USA) were directly bonded to the labial surfaces of the teeth following the manufacturers bonding protocol for each of the materials. Afterwards all teeth were submitted to a thermo cycling procedure for 1,000 cycles at 5 degrees $C$ and 55 degrees $C$ with a dwell time of $20 \mathrm{~s}$. After the accelerated staining/bleaching procedures, all samples were submitted to a Shear-Bond Strength test. The Machine of choice used for SBS testing was the LR5KPlus 5kNUniversal Materials Testing Machine from Lloyd (West Sussex, UK). In order to test the SBS, without touching the enamel and while using a crosshead speed of $1 \mathrm{~mm} / \mathrm{min}$, a knife-edged shearing blade was positioned parallel to both the labial surface and the bracket interface to allow the transmission of the force in the occlusogingival direction. The force applied at the time of fracture was recorded in Newton's (N) and converted to megapascals (Mpa) dividing the force by the bracket base area, which according to the manufacturer have a mesh base area of $0.115 \mathrm{~cm}^{2}$.

Statistical analysis StudentT-tests, Chi-square and Anova tests were used for results interpretation. T tests emphasize the differences that exist compared to controls, while chi square test had shown significant differences between materials. Results were considered significant for $t<10$. The statistical package Origin Pro 8 SRO (OriginLab Corporation 2007, Northampton MA 01060, USA) was used for data analyses.

\section{Results and discussions}

\section{Cell viability}

As we can see in figure 1 , the controls exhibited a significant proliferation at $72 \mathrm{~h}(\mathrm{p}<0,001)$. In the treated cultures, there were significant cell viability differences among the tested materials.

Exposure of HUVEC cultures to both resin hybrid glassionomers (Fuji Ortho LC and Experimental) for $24 \mathrm{~h}$ greatly reduced the viability rate $(t=64.56 . t=56.93)$, whereas the resin-based materials treated cultures (Opal Bond, Light-bond and Vertise) showed better viabilities $(t=4.76, t=3.29, t=3.15)$, with Light-bond being the most cytotoxic resin base adhesive, compared to Opal Bond and to Vertise. After $72 \mathrm{~h}$ all of the tested adhesive materials reduced HUVEC viability when compared with the control group, without significant differences amongst them. 


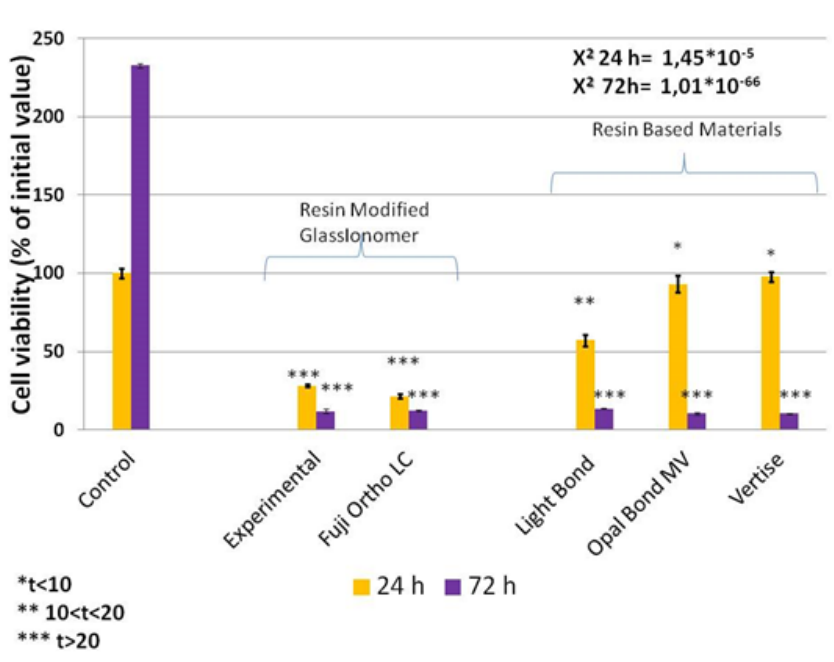

Fig. 1. Graph showing cell proliferation after $24 \mathrm{~h}$ and $72 \mathrm{~h}$ exposure to bonding materials (vs. untreated HUVECs); mean values and standard deviations $(n=3)$ are shown; all materials induced decreased viability, which was significant after $24 \mathrm{~h}$, the effect was more evident for resin modified glassionomers; after $72 \mathrm{~h}$ all treated cells exhibited comparable values for viability (*t <

10 , viability of treated cells versus control, Student's t-test)

\section{Cell death induction}

Exposure to the Experimental material increased the apoptotic, annexin V positive cells, compared to controls at both time points ( $\mathrm{t}=96.26$ at $24 \mathrm{~h}$ and $\mathrm{t}=110.6$ at $72 \mathrm{~h}$ ). The necrotic PI positive cells were not increased with $24 \mathrm{~h}$ of exposure ( $t=9.61)$, but were greatly increased after 72 $h$ ( $t=156.37)$ (fig. 2, 3 ). The same effect was observed with the other resin hybrid glassionomer (Fuji Ortho LC), but the intensity was lower at $72 \mathrm{~h}$ of exposure $(\mathrm{t}=76.61$ at $24 \mathrm{~h}$ and $\mathrm{t}=34.7$ at $72 \mathrm{~h}$, for annexin V positive cells and $\mathrm{t}=101.9$ at $24 \mathrm{~h}$ and $\mathrm{t}=62.66$ at $72 \mathrm{~h}$, for PI positive cells) (fig. 2,3). The resin base adhesives Light-bond, Opal- bond and Vertise showed a different pattern. They all increased the necrotic, PI positive cells, in a time dependent manner ( $t=20.79$ for Light-bond, $t=17.24$ for Opal-bond and $t=63.160$ for Vertise at $24 \mathrm{~h}$ and $\mathrm{t}=95.66$ for Light-bond, $t=96.75$ for Opal-bond and $t=44.41$ for Vertise at $72 \mathrm{~h}$ ). The apoptotic annexin $V$ positive cells were only increased at $72 \mathrm{~h}$ and the effect was more important for Opal Bond $(t=97.76) .72 \mathrm{~h}$ reading revealed an increased ratio of necrotic cells for the Experimental material, followed by Light Bond and Opal Bond. These findings suggest that the cell death mechanism is different for the two classes of materials studied. The resin hybrid glassionomers mainly induced apoptosis, while the resin base adhesives induced necrotic death. The experimental material increased necrotic death after $72 \mathrm{~h}$ of exposure, but this was not the primary mechanism of cell death, and in our opinion it occurred due to lack of stability of the compound in the culture media, with long term exposure, which allowed toxic substances to accumulate.

The control culture displayed a regular morphology with dense cells, elongated and cobblestone-shaped in appearance, with long slender elongations and numerous mitoses.

With the resin based tested materials and the Fuji-Ortho, the culture appeared less dense than the control, but normal cells were available. There were only a few rounded or detached cells. While materials led to enlargement of the intercellular space, the cells kept their cobblestone shape. Some cells were in apoptosis showing degenerative effects such as loss of cellular elongations, vacuolization

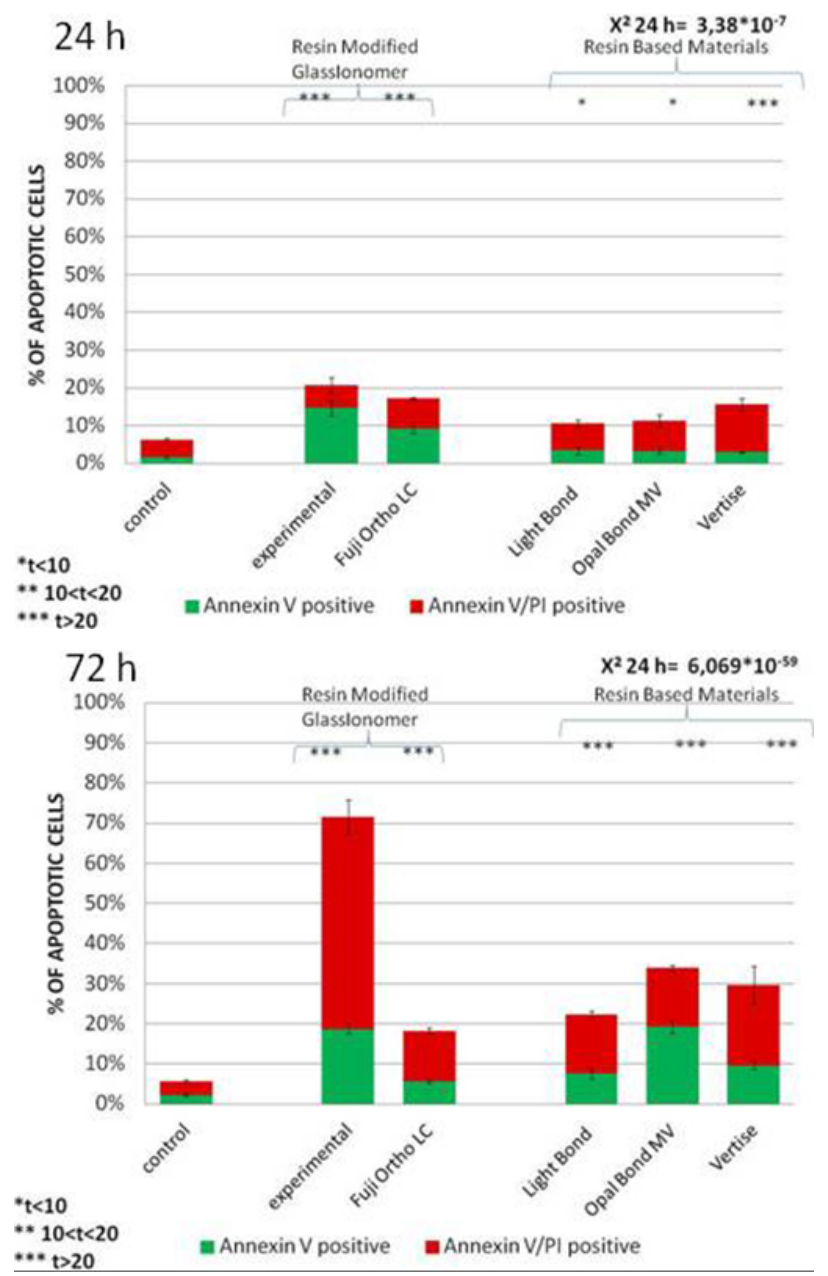

Fig 2. Assessment of apoptosis by positive signal for Annexin $V$ and $\mathrm{PI}$ in control HUVECs versus treated with dental materials for 24 , respectively 72 hours. The percent of apoptotic cells identified as Annexin $\mathrm{V}(+)$ (green) shows a significant increase in all treated cultures versus controls after $24 \mathrm{~h}$ of exposure. The percent of apoptotic cells identified as Annexin V (+) (green) and late apoptotic/necrotic cells, identified as PI $(+)$ (red) shows a significant increase in all treated cultures versus controls after $72 \mathrm{~h}$ of exposure, the effect is more important for resin based materials and experimental bonding material $(t<10$, chi square test was used for statistical comparison). Each bar represents mean and standard deviation $(n=3)$

of the cytoplasm, loss of surface adherence and polimorphysm.

Most cells exposed to media containing the Experimental materials were significantly retracted, rounded or float in the culture medium, and also an increased intercellular space was noted.

Caspase 3 is the common pathway for the apoptotic cell death. Measurement of the caspase 3 was done through ELISA technique and is a marker of early apoptotic cell death. The control cultures exhibited no significant difference with time in caspase 3 levels. Experimental and Fuji Ortho LC materials significantly increased caspase 3 at $24 \mathrm{~h}(\mathrm{t}=15.25, \mathrm{t}=20.21)$. This effect was not as important at $72 \mathrm{~h}(\mathrm{t}=11.16, \mathrm{t}=6.65)$. Interestingly, the Experimental material has shown a lower production of caspase 3 when compared to Fuji Ortho LC. This is probably due to the decrease of the overall cell number and thus the production of caspase 3. This pattern of caspase 3 induced by the two resin hybrid glassionomers, is consistent with annexin V/ PI staining results and sustains the idea that the main mechanism of cell death in the cultures is increased apoptosis. 


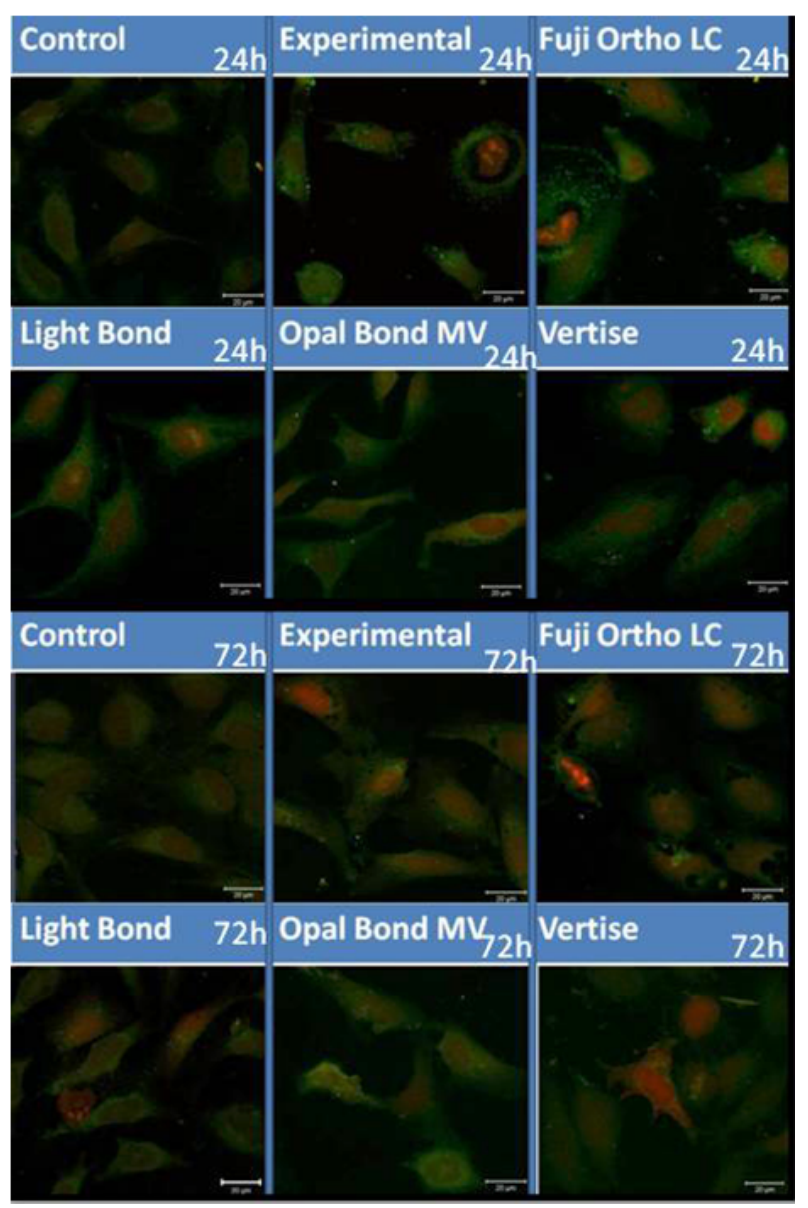

Fig. 3. Assessment of apoptosis by positive signal for Annexin $\mathrm{V}$ and $\mathrm{PI}$ in control and treated HUVECs following exposure to bonding materials for $24 \mathrm{~h}$. Confocal microscopy images of HUVECs [double staining with annexinV (green) and PI (red)] vs. controls. Colocalized signal displays yellow color. Controls show negative for annexinV/PI staining; in HUVECs treated with bonding materials, there is evidence for apoptotic cells (green -annexin $\mathrm{V}$ positive) and even a few PI positive cells, especially for Vertise at $24 \mathrm{~h}$ reading. The number of apoptotic and/or necrotic cells is

increased with time elapsed, at $72 \mathrm{~h}$ all treated cultures show images of double stained positive cells. Original magnification 63x

The other three resin based materials Light-bond, Opalbond and Vertise showed lower caspase 3 values at $24 \mathrm{~h}$ $(\mathrm{t}=7.39, \mathrm{t}=22.54, \mathrm{t}=20.56)$, but it increased with time exposure $(t=12.27, t=11.58, t=2.67)$. Opal Bond had the most important proapoptotic effect. This is also consistent with the annexin V/PI findings which showed increased apoptosis with time exposure. These findings, suggest that the main mechanism of cell death induced by the resin based materials materials is in fact necrosis, and only after longer time, e.g. $72 \mathrm{~h}$ of exposure, the cell death is partly apoptotic.

TNF $\underline{\alpha}$ is a marker of inflammatory reaction. It was measured from cell culture supernatants through ELISA technique. The control cultures showed a decrease with time. Experimental material did not significantly modify the TNF $\alpha$, compared to controls at $24 \mathrm{~h}$ of exposure $(t=0.74)$. However, longer exposure greatly increased the level of TNF $\alpha$ (approximately $8 x, t=117.55$ ). The same pattern of TNF $\alpha$ modification was seen in the case of Vertise, with a slight increase after $24 h(t=7.13)$, then an important increase at $72 \mathrm{~h}(\mathrm{t}=71.28)$. When HUVEC cultures were exposed to the other three materials, Fuji Ortho LC, Light Bond and Opal Bond MV there was a

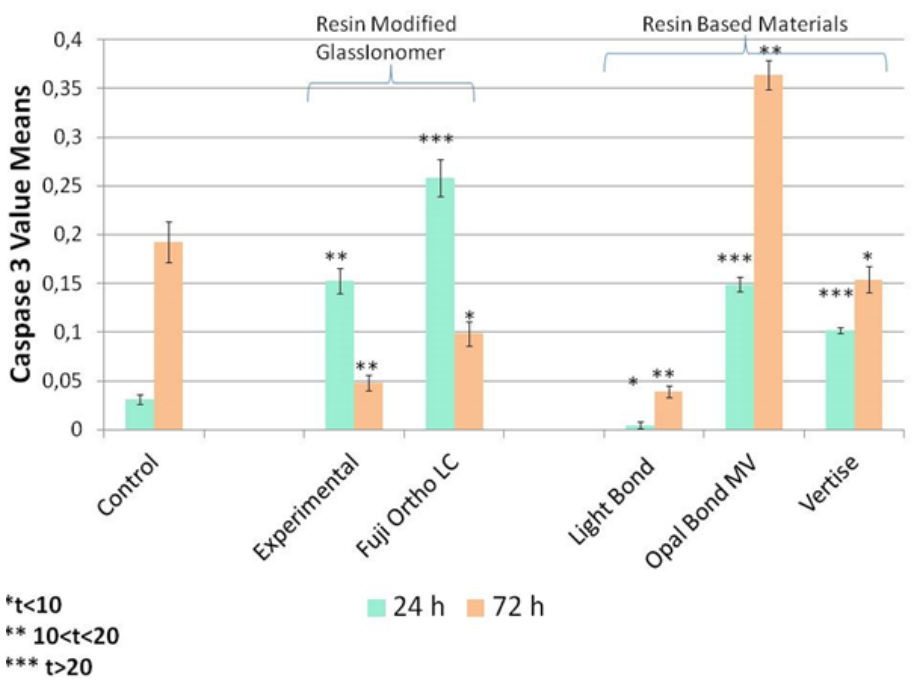

Fig. 4. ELISA measurement of active caspase $3(\mathrm{ng} / \mathrm{ml})$ in HUVECS treated with bonding materials (vs. control) after $24 \mathrm{~h}$ and $72 \mathrm{~h}$ of exposure. For resin modified glassionomers, increased caspase 3 at $24 \mathrm{~h}$ (vs. control) was observed, followed by a gradual decline; at 72 $\mathrm{h}$ the caspase 3 activity was significantly decreased compared to the level observed in controls; resin based materials increased caspase 3 levels with time exposure, the maximum increase was exhibited by Opal Bond MW. Student's t-test was used for statistical comparison.

Each bar represents mean and standard deviation $(n=3)$

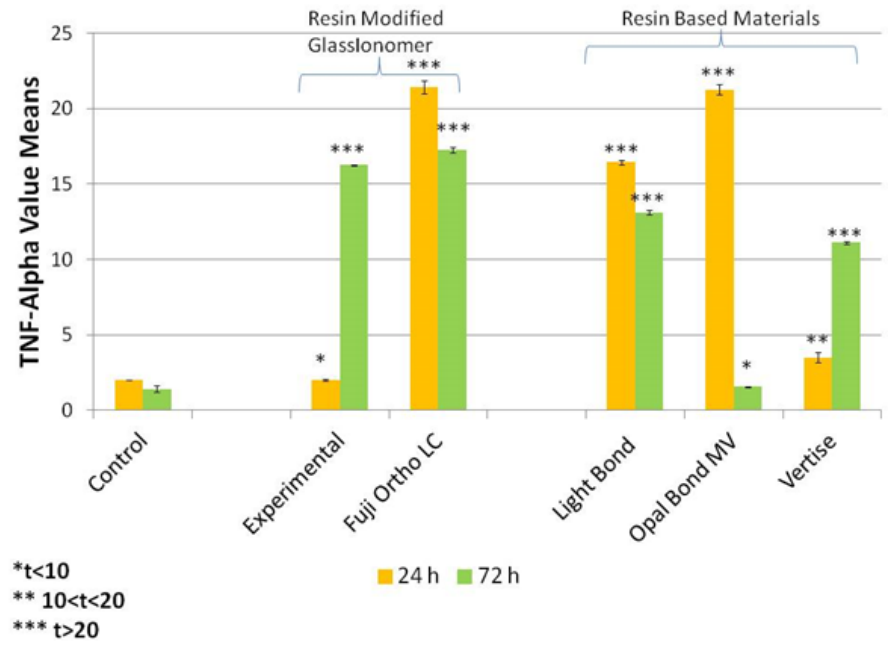

Fig. 5. ELISA measurement of TNF-alpha levels $(\mathrm{pg} / \mathrm{ml})$ in HUVECs treated with bonding materials (vs. control) after $24 \mathrm{~h}$ and $72 \mathrm{~h}$ of exposure. All bonding materials increased TNF-alpha at $24 \mathrm{~h}$ (vs. control); HUVECs treated with experimental bonding material exhibited the lowest level; this was followed by a gradual decline for Fuji Ortho LC, Light Bond, Opal Bond while the others (Experimental and Vertise) showed a strong increase; at $72 \mathrm{~h}$ the TNF-alpha was still significantly increased compared to the level observed in controls. Student's t-test was used for statistical comparison. Each bar represents mean and standard deviation $(n=3)$

different effect, with an initial increase at $24 \mathrm{~h}(\mathrm{t}=79.32 \mathrm{for}$ Fuji Ortho $L C, t=159.233$ for Light Bond, $t=98.16$ for Bond MV), followed by a slight decrease in the case of Fuji Ortho LC ( $\mathrm{t}=77.21)$ and Light Bond ( $\mathrm{t}=91.88)$ at $72 \mathrm{~h}$. Opal bond MV treated cultures showed levels of TNF $\alpha$ comparable with those of controls at $72 \mathrm{~h}(\mathrm{t}=11.20)$.

\section{Shear Bond Strength testing}

There was no statistical difference in the mean SBS amongst the commercial resin material groups (Opal Bond MV and Light Bond). However there was an important difference $(p=0.0236)$ between them and Vertise, the latter having shown a very low resistance to shear-bond stress. 


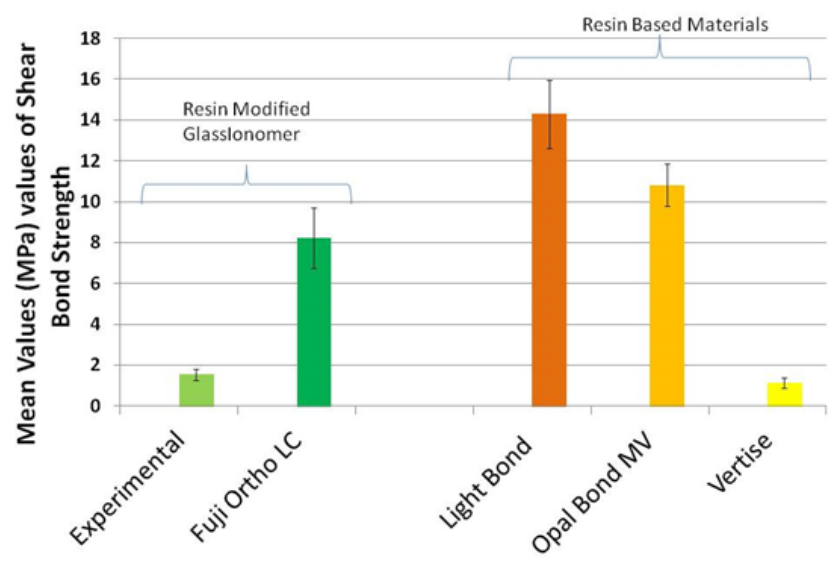

Fig. 6. Shear Bond Strength Testing results showing a clinically adequate performance for all commercial materials with orthodontic indication. Light Bond exhibited an average strength to shear bond stress above 14MPa, followed by Opal Bond MV with $11 \mathrm{MPa}$ and the resin modified glass-ionomer Fuji Ortho LC. The least performant was Vertise (1MPa) and the Experimental resinmodified glass-ionomer also under $2 \mathrm{Mpa}$

There were also significant differences between the glassionomer type adhesives, the Experimental material performed in a less satisfactory manner $(p=0.0048)$. The Chi-squared test $\chi^{2}=109.673$ revealed important differences between SBS values corresponding to the different materials tested $(p<0.0005)$.

It is important to evaluate the biocompatibility of the orthodontic bonding adhesives, because these materials are located proximate to the periodontal tissue and alveolar bone. Substances released from orthodontic composites may cause a reaction (inflammation or necrosis) to adjacent tissues, such as oral mucosa, gingiva or alveolar bone. There are different ways in which materials may influence the health of soft tissues: by delivering watersoluble components into the saliva and the oral cavity as well as by directly interacting with adjacent tissues [11]. The orthodontic adhesives were tested in vitro, on cell cultures to simulate the clinical setting as closely as possible. HUVEC cells and gingival fibroblasts have previously been shown to have similar cytotoxicity levels. Consequently, HUVEC cells make a useful screening model for in vitro toxicity testing of dental materials [12]. Because of its excellent reproducibility, the HUVEC cell line was preferred to primary gingival fibroblasts. However, in vitro cytotoxicity testing conditions cannot perfectly simulate the intra-oral conditions because in the last situations there are enzymes able to neutralize some of the side effects. Different orthodontic adhesives were found to have varying toxicity levels in vitro and some were shown to lose their toxicity more rapidly than others. A study of dental composites found that a chemically cured composite is significantly more cytotoxic than a light-cured composite of similar composition [1, 12]. Dental composite cytotoxicity has previously been shown to decrease significantly after 7 days of pre-incubation $[13,14]$. Therefore, fresh specimens have shown an increased cytotoxicity compared to other studies that used preincubated materials for 7 days.

In our study we measured cell viability as an indicator of the overall cytotoxic effect. All tested materials were found to be cytotoxic. The experimental material showed a significantly higher cytotoxicity, probably due to a less stable formula and needs further testing.

In our study, we used light-cured, fresh samples, which might partially explain the high cytotoxicity levels. Also, the amount of material contained in each sample was significantly higher than the amount needed to bond one bracket. We further studied the mechanism of cell death induction and inflammatory reaction since these can directly influence the clinical outcome of the therapy. Interestingly, there were significant differences in the evolution of cytotoxicity with time exposure as well as the cell death mechanism, related to the chemical structure of the compounds. The Resin-hybrid glassionomer base materials showed an increased cytotoxic effect at the 24 hour reading when compared to the resin-based but they all exhibited similar levels at the $72 \mathrm{~h}$ reading. Based on annexin V/PI staining and caspase 3 levels, the main mechanism of cell death in the cultures treated with the resin hybrid glassionomers is increased apoptosis, while the resin based materials induced mainly necrotic cell death. This can be of clinical importance since necrosis leads to cell desintegration and spilling of the cells content, including lysosomal enzymes directly in the intercellular space. This damages the intercellular matrix and leads to an inflamatory reaction in the surrounding tissue.

Tumor necrosis factor (TNF) represents a proinflammatory cytokine that stimulate a number of events like: the induction of adhesion molecules and other mediators that facilitate and amplify the inflammatory response, the stimulation of matrix metalloproteinase, and bone resorption. Elevated TNF- $\alpha$ levels in saliva were correlated with the severity of oral lichenus planus, a chronic inflammatory disease of the oral mucosa as well as with squamous cell carcinoma. Even though all tested materials increased TNF $\alpha$ production from treated HUVEC cells, the dynamic of the increase was strongly influenced by the chemical composition. Resin-hybrid glassionomers increased TNF $\alpha$ in a time dependent manner, while the resin based materials increased TNF $\alpha$ levels at $24 h$, afterwards, the inflammatory marker decreased to almost control values for Opal Bond [ 10].

There are numerous factors influencing bond strength between adhesives and tooth structures, which makes interpretation of the results difficult. To obtain meaningful information about the performance of adhesives, fracture analysis is a condition sine qua non. Although shear bond strength was different amongst the studied materials, additional fracture analysis on teeth can give similar qualitative information.

All tested adhesives had a better behaviour than the tested self etch adhesive (Vertise). We observed a better adhesion performance with commercial brackets adhesives than with the experimental one. This result is probably due to a relatively less degree of conversion of the polymerized composite and adhesives in the experimental materials. Moreover, the statistical analysis suggested that the adhesive interface resulting between the tooth and the resin-based commercial adhesives had a better behavior than resin-based glassionomers.

With the limitations of a test in which many variables were not included, such as, for example, the aging of the samples, this study, without pointing out the single values, gives us the possibility to compare different adhesive's performances in simulated clinical conditions.

\section{Conclusions}

On the basis of collected data and among tested adhesives, the Light Bond adhesive confirmed the best characteristics between operating difficulties and the gained adhesion.

When looking at the in vitro study, Light Bond, but also Opal Bond had the lowest citotoxic effects. However, 
further studies using different test methods are needed for these materials. Research efforts should focus on assessing long-term biologic effects of orthodontic adhesives.

Acknowledgements: The present paper represents the results of a team work, funded by Iuliu Hatieganu University of Medicine and Pharmacy, Cluj-Napoca, through the internal research grant nr. 4944/ 15/08.03.2016 and it is part of the PhD-Thesis Investigations of the impact of orthodontic treatment upon dento-facial esthetics of the first author.

\section{References}

1.AHRARI F, AFSHARI JT, POOSTI M, BROOK A, Cytotoxicity of orthodontic bonding adhesive resins on human oral fibroblasts Eur J Orthodont 201032 (6): 688-692

2.ESTLANDER T, RAJ ANIEMI R, J OLANKI R. Hand dermatitis in dental technicians. Contact Dermatitis 1984;10:201-205

3.MUNKSGAARD EC, HANSEN EK, ENGEN T, HOLM U. Self-reported occupational dermatological reactions among Danish dentists. Eur J Oral Sci 1996; 104:396-402

4.GEUKENS S,GOOSSENS A. Occupational contact allergy to (meth)acrylates. Contact Dermatitis 2001;44:153-159

5.Huang TH , Liao PH., Li HY, Ding SJ , Yen M, Kao CT Orthodontic Adhesives Induce Human Gingival Fibroblast Toxicity and Inflammation. Angle Orthod, 2008, 78, 3. 510-516.

6.SHARMA P, VALIATHAN A, ARORA A, AGARWAL S. A comparative evaluation of the retention of metallic brackets bonded with resinmodified glass ionomer cement under different enamel preparations: A pilot study. Contemp Clin Dent. $2013,4(2): 140-6$
7.VAN ENGELAND N, NIELAND LJW, RAMAEKERS FCS, SCHUTTE B, REUTELINGSPERGER CPM. Annexin V-affinity assay. A review on an apoptosis detection system based on phosphtidylserine exposure. Cytometry 1998;31:1-9.

8.GRAVES DT. The Contribution of Interleukin-1 and Tumor Necrosis Factor to Periodontal Tissue Destruction, J Periodontol 2003, 74( 3), 391-401

9.PEZELJ -RIBARIC S, PRSO IB, ABRAM M, GLAZAR I, BRUMINI G, SIMUNOVIC-SOSKIC M. Salivary levels of tumor necrosis factor-alpha in oral lichen planus. Mediat Inflamm. 2004 13(2):131-3

10.JIN L., STURGIS EM. , ZHANG Y, HUANG Z., SONG X, LI C, WEI Q AND LI G Association of tumor necrosis factor-al pha promoter variants with risk of HPV-associated oral squamous cell carcinoma Mol Cancer 2013, 12:80

11.YALCIN M, ULKER M, ULKER E, SENGUN A. Evaluation of cytotoxicity of six different flowable composites with the methyl tetrazolium test method. Eur J Gen Dent 2013;2:292-5

12.SCHEDLE A, FRANZ A, RAUSCH-FAN X, SPITTLER A, LUCAS T, SAMORAPOOMPICHIT P, SPERR W, BOLTZ-NITULESCU G. CytotoxiC effects of dental composites, adhesive substances, compomers and cements. Dent Mater 1998;14:429-440

13.FRANZ A, KONIG F, ANGLMAYER M, RAUSCH-FAN X, GILLE G, RAUSCH WD, LUCAS T, SPERR W, SCHEDLE A. Cytotoxic effects of packable and nonpackable dental composites. Dent Mater 2003;19:382392.

14.FRANZ A, KONIG F, LUCAS T, WATTS DC, Schedle A. Cytotoxic effects of dental bonding substances as a function of degree of conversion. Dent Mater 2009;25:232-239

Manuscript received: 31.10 .2017 